$19^{\text {th }}$ European Symposium on Computer Aided Process Engineering - ESCAPE19

J. Jeżowski and J. Thullie (Editors)

(C) 2009 Elsevier B.V./Ltd. All rights reserved.

\title{
Variance of Estimates in Dynamic Data Reconciliation
}

\author{
Christophe Ullrich, Georges Heyen and Carine Gerkens
}

University of Liège, Lassc, Allée de la Chimie 17, B6A, Sart-Tilman, Liège B-4000, Belgium, cullrich@ulg.ac.be

\begin{abstract}
The method previously proposed to estimate the uncertainty of validated variables in steady state data reconciliation has been extended to dynamic data reconciliation.

The approach used in this article to estimate a posteriori variances in the case of dynamic date validation is based on the one described in [2] for the stationary case. Orthogonal collocations are used to discretise ODE. Results are presented for an adiabatic reactor with first order kinetic.
\end{abstract}

Keywords: dynamic data reconciliation, a posteriori variances, orthogonal collocations

\section{Introduction}

Efficient process monitoring is a key issue in plant operation, since measurement errors are always present. To address this issue, data validation is nowadays routinely performed for steady state processes, but dynamic systems still present some challenges. Data validation uses measurement redundancy and model constraints to reduce measurement uncertainty and to calculate non measured state variables of the system. A posteriori variance for validated variables compared to raw measurements can be calculated for linear or linearized steady state systems. Several methods are enable to solve the 
dynamic data reconciliation problem [2]. We use NLP technique and orthogonal collocation [3] to discretize the ODE systems, as described in [1].

\section{Estimation of a posteriori variances}

The algorithm uses moving horizon is used to limit the size of the optimization problem. This moving horizon is described by the following figure.

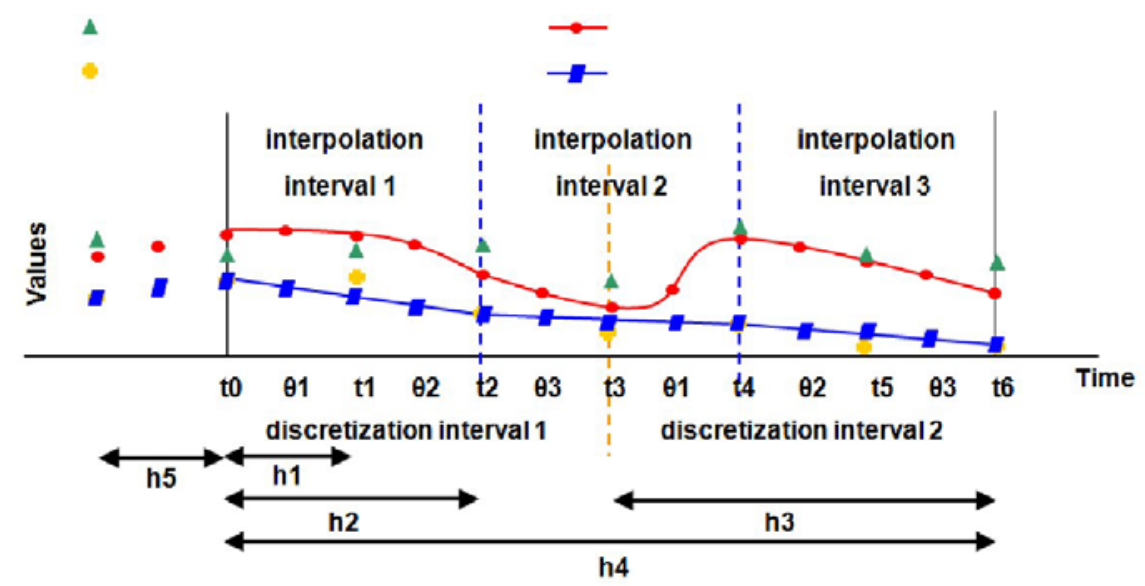

The validation window is defined by five parameters:

- $\mathrm{h}_{1}$ : measurement frequency

- $\mathrm{h}_{2}$ : size of the interpolation of the input variables

- $\mathrm{h}_{3}$ : discretization interval of the differential state variables

- $\mathrm{h}_{4}$ : size of the moving window

- $\mathrm{h}_{5}$ : the move of the window after optimization

In the case of orthogonal collocations, the objective function is:

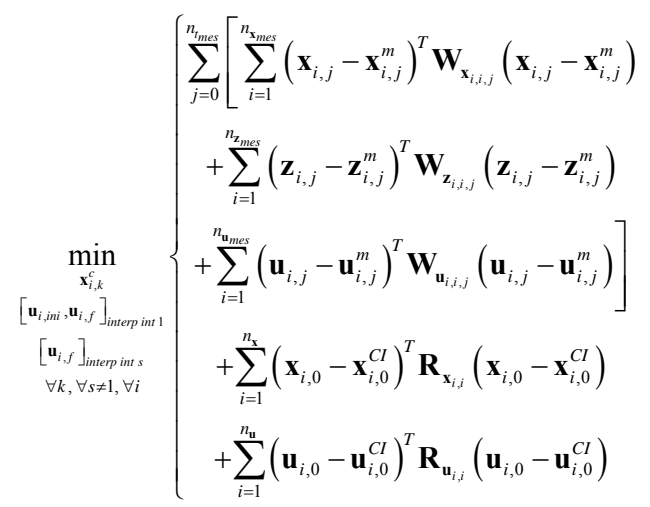




\section{Variance of estimates in dynamic data reconciliation}

With

- $\quad \mathbf{x}$ : the vector of differential state variables;

- $\quad$ : the vector of algebraic variables;

- $\quad \mathbf{u}$ : the vector of input variables.

This objective function is submitted to five types of constraints:

- the link equations: they are algebraic relations between all process variables. Those constraints have to be satisfied as well at the measurements times as at the collocations nodes:

$$
\mathbf{A}=f\left(t_{j}, \mathbf{x}, \mathbf{z}, \mathbf{u}\right)=\mathbf{0} \quad \forall t_{j} \quad \mathbf{A}^{c}=f^{c}\left(\theta_{k}, \mathbf{x}^{c}, \mathbf{z}^{c}, \mathbf{u}^{c}\right)=\mathbf{0} \quad \forall \theta_{k}
$$

- the relations between the differential state variables and the Lagrange interpolation polynomials at all measurement times of the moving horizon except at the initial times of the discretization intervals $t_{\mathrm{CI}}$ :

$$
\mathbf{B}=\mathbf{x}_{i, j}-\sum_{k=0}^{n_{\theta}} \mathbf{l}_{k}\left(t_{j}\right) \mathbf{x}_{i, k}^{c}=\mathbf{0} \quad \forall i, \forall t_{j} \neq t_{C I}
$$

- the linear interpolations of the values of input variables between times $t_{\text {ini }}$ and $t_{\mathrm{f}}$ of the interpolation horizon at the other times of that horizon:

$$
\begin{array}{ll}
\mathbf{C}=\mathbf{u}_{i, j}-\mathbf{u}_{i, t_{\text {ini }}}-\frac{t_{j}-t_{\text {ini }}}{t_{f}-t_{i n i}}\left(\mathbf{u}_{i, t_{f}}-\mathbf{u}_{i, t_{\text {tin }}}\right)=\mathbf{0} & \forall i, \forall t_{j} \neq t_{i n i}, t_{f} \\
\mathbf{C}^{c}=\mathbf{u}_{i, k}^{c}-\mathbf{u}_{i, t_{\text {ini }}}-\frac{\theta_{k}-t_{\text {ini }}}{t_{f}-t_{i n i}}\left(\mathbf{u}_{i, t_{f}}-\mathbf{u}_{i, t_{\text {tii }}}\right)=\mathbf{0} & \forall i, \forall \theta_{k}
\end{array}
$$

- the residuals of the differential state equations at all collocation nodes:

$$
\mathbf{D}=\sum_{s=0}^{n_{g}} \mathbf{1}_{s}\left(\theta_{k}\right) \mathbf{x}_{i, \theta_{s}}^{c}-g\left(\theta_{k}, \mathbf{x}_{k}^{c}, \mathbf{z}_{k}^{c}, \mathbf{u}_{k}^{c}\right)=\mathbf{0} \quad \forall i, \forall \theta_{k} \neq t_{i n i}
$$

- the continuity constraints of the differential state variables between two discretization intervals:

$$
\mathbf{E}=\left[\sum_{k=0}^{n_{\theta}} \mathbf{l}_{k}\left(t_{f}\right) \mathbf{x}_{i, k}^{c}\right]_{t_{f}, \text { discr int } q-1}-\left[\sum_{k=0}^{n_{\theta}} \mathbf{l}_{k}\left(t_{i n i}\right) \mathbf{x}_{i, k}^{c}\right]_{t_{i n} \text {, discr int } q}=\mathbf{0} \quad \forall i
$$

This constrained problem can be transformed into an unconstrained problem using Lagrange formulation. The necessary condition for optimality is expressed by setting to 0 the gradient of the Lagrangian. By linearising the equation system as shown in [2], one obtains a linear relation between validated variables and measurements:

$$
\begin{aligned}
& \mathbf{M}\left(\begin{array}{llllllllllllll}
x & z & u & x^{c} & z^{c} & u^{c} & \Lambda^{A} & \Lambda^{A^{c}} & \Lambda^{B} & \Lambda^{C} & \Lambda^{C^{c}} & \Lambda^{D} & \Lambda^{E}
\end{array}\right)^{T} \\
& =\left(\begin{array}{lllllllllllll}
P_{x} x^{m} & P_{z} z^{m} & P_{u} u^{m} & 0 & 0 & 0 & -F & -F^{c} & 0 & 0 & 0 & -G & 0
\end{array}\right)^{T}
\end{aligned}
$$


With $F, F^{c}$ and $G$ the constant terms of the linear aproximation of the constraints.

One obtains the sensitivity matrix $M$ which is the Jacobian matrix of the equation system:

$$
\begin{aligned}
\mathbf{M} & =\left(\begin{array}{cc}
\mathcal{P} & \mathcal{E} \\
\mathcal{E}^{T} & 0
\end{array}\right) \\
\mathcal{P} & =\left(\begin{array}{cccccc}
\mathbf{P}_{\mathbf{x}} & \mathbf{0} & \mathbf{0} & \mathbf{0} & \mathbf{0} & \mathbf{0} \\
\mathbf{0} & \mathbf{P}_{\mathbf{z}} & \mathbf{0} & \mathbf{0} & \mathbf{0} & \mathbf{0} \\
\mathbf{0} & \mathbf{0} & \mathbf{P}_{\mathbf{u}} & \mathbf{0} & \mathbf{0} & \mathbf{0} \\
\mathbf{0} & \mathbf{0} & \mathbf{0} & \mathbf{0} & \mathbf{0} & \mathbf{0} \\
\mathbf{0} & \mathbf{0} & \mathbf{0} & \mathbf{0} & \mathbf{0} & \mathbf{0} \\
\mathbf{0} & \mathbf{0} & \mathbf{0} & \mathbf{0} & \mathbf{0} & \mathbf{0}
\end{array}\right) \quad \mathcal{E}=\left(\begin{array}{ccccccc}
\frac{\partial \mathbf{A}}{\partial \mathbf{x}} & \frac{\partial \mathbf{A}}{\partial \mathbf{z}} & \frac{\partial \mathbf{A}}{\partial \mathbf{u}} & \mathbf{0} & \mathbf{0} & \mathbf{0} \\
\mathbf{0} & \mathbf{0} & \mathbf{0} & \frac{\partial \mathbf{A}^{c}}{\partial \mathbf{x}^{c}} & \frac{\partial \mathbf{A}^{c}}{\partial \mathbf{z}^{c}} & \frac{\partial \mathbf{A}^{c}}{\partial \mathbf{u}^{c}} \\
\frac{\partial \mathbf{B}}{\partial \mathbf{x}} & \mathbf{0} & \mathbf{0} & \frac{\partial \mathbf{B}}{\partial \mathbf{x}^{c}} & \mathbf{0} & \mathbf{0} \\
\mathbf{0} & \mathbf{0} & \frac{\partial \mathbf{C}}{\partial \mathbf{u}} & \mathbf{0} & \mathbf{0} & \mathbf{0} \\
\mathbf{P} & =\mathbf{W}_{\mathbf{x}}+\mathbf{R}_{\mathbf{x}} & & \\
\mathbf{P}_{\mathbf{z}}=\mathbf{W}_{\mathbf{z}} & & & \\
\mathbf{P}_{\mathbf{u}}=\mathbf{W}_{\mathbf{u}}+\mathbf{R}_{\mathbf{u}} & & \frac{\partial \mathbf{C}^{c}}{\partial \mathbf{u}} & \mathbf{0} & \mathbf{0} & \frac{\partial \mathbf{C}^{c}}{\partial \mathbf{u}^{c}} \\
\mathbf{0} & \mathbf{0} & \mathbf{0} & \frac{\partial \mathbf{D}}{\partial \mathbf{x}^{c}} & \frac{\partial \mathbf{D}}{\partial \mathbf{z}^{c}} & \frac{\partial \mathbf{D}}{\partial \mathbf{u}^{c}} \\
\mathbf{0} & \mathbf{0} & \mathbf{0} & \frac{\partial \mathbf{E}}{\partial \mathbf{x}^{c}} & \mathbf{0} & \mathbf{0}
\end{array}\right)
\end{aligned}
$$

As for the stationary estimation [2], posteriori variances can be deduced from those last formulas:

$$
\begin{aligned}
\operatorname{var}\left(\mathbf{x}_{k}\right) & =\sum_{i=1}^{N_{x}}\left[\mathbf{M}_{k, i}^{-1} \mathbf{P}_{\mathbf{x}_{i}}\right]^{2} \operatorname{var}\left(\mathbf{x}_{i}^{m}\right)+\sum_{i=1}^{N_{z}}\left[\mathbf{M}_{k, i+N_{x}}^{-1} \mathbf{P}_{\mathbf{z}_{i}}\right]^{2} \operatorname{var}\left(\mathbf{z}_{i}^{m}\right) \\
& +\sum_{i=1}^{N_{u}}\left[\mathbf{M}_{k, i+N_{x}+N_{z}}^{-1} \mathbf{P}_{\mathbf{u}_{i}}\right]^{2} \operatorname{var}\left(\mathbf{u}_{i}^{m}\right)
\end{aligned}
$$

Similar equations can be written for input and algebraic variables.

\section{Case study: an adiabatic reactor with first order kinetic}

This reactor with first order kinetic is defined by the following differential state equation:

$\frac{d C A}{d t}=\frac{F}{V}\left(C A_{i}-C A\right)-k C A$

$\mathrm{CA}$ is the state variables while $\mathrm{F}$ and $\mathrm{CA}_{\mathrm{i}}$ are the inputs. The problem has no algebraic variable. The kinetic constant $\mathrm{k}$ is defined as a constant of the optimization problem; so, it can not be optimized. 
The parameters of the window have been chosen as follow: $h_{1}=1, h_{2}=4, h_{3}=4$, $\mathrm{h}_{4}=49$ and $\mathrm{h}_{5}=2$. The Lagrange interpolation polynomials are of the second order. The process is subject to several inputs changes of the form:

$\frac{d x}{d t}=K\left(x_{S P}-x\right)$

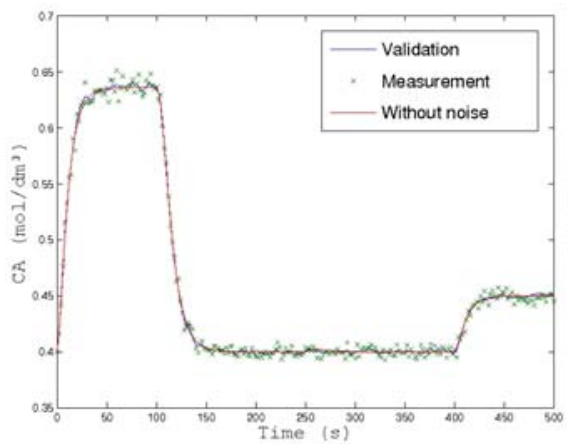

Figure 1: Concentration profile

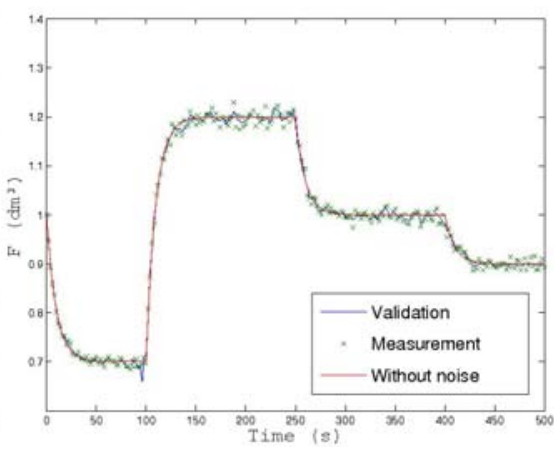

Figure 2: Feed flowrate profile

As can be seen on figure 1 and 2, for the concentration and the feed flowrate profiles respectively, the validation allows to reduce the noise and the changes in the profiles are very well followed by the validated values.
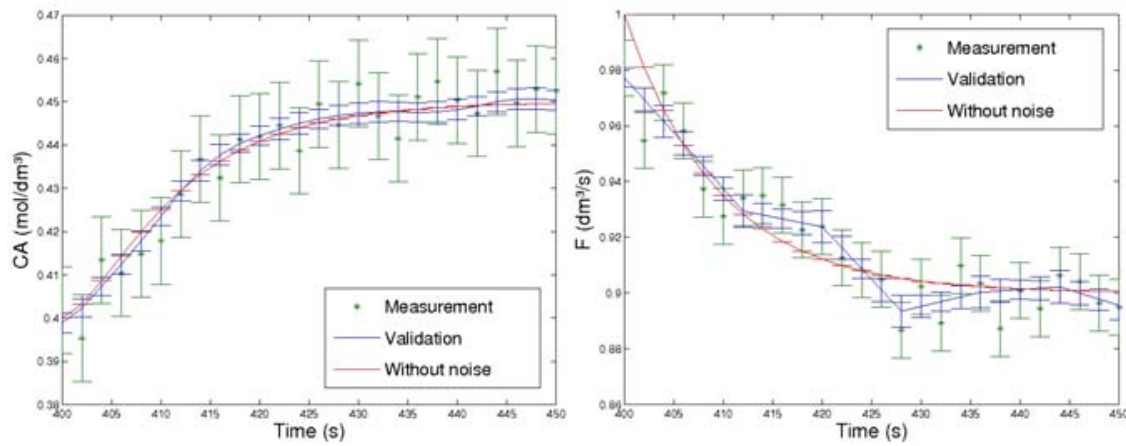

Figures 3 and 4: Uncertainties comparison for concentration and feed flowrate

Figures 3 and 4 show the standard deviation of the measurements and the validated values for the concentration and the feed flowrate respectively. The variances are reduced as well for state variables as for the inputs.

For the state variable, one has the following results:

\begin{tabular}{|c|c|c|c|}
\hline Times & $\begin{array}{c}\text { A priori } \\
\text { variances }\end{array}$ & $\begin{array}{c}\text { A posteriori } \\
\text { variances }\end{array}$ & $\begin{array}{c}\text { Reduction } \\
\text { factor }\end{array}$ \\
\hline 360 & 0.01 & 0.0047 & 2.1 \\
\hline 380 & 0.01 & 0.0021 & 4.8 \\
\hline
\end{tabular}


C. Ullrich et al.

\begin{tabular}{|l|l|l|l|}
\hline 400 & 0.01 & 0.0023 & 4.3 \\
\hline 420 & 0.01 & 0.0022 & 4.5 \\
\hline 440 & 0.01 & 0.0024 & 4.2 \\
\hline
\end{tabular}

The variance reduction is similar for all measurement times of the validation window excepting for the first times for which it is less important.

For the incoming flowrate, one has the following results:

\begin{tabular}{|c|c|c|c|}
\hline Times & $\begin{array}{c}\text { A priori } \\
\text { variances }\end{array}$ & $\begin{array}{c}\text { A posteriori } \\
\text { variances }\end{array}$ & $\begin{array}{c}\text { Reduction } \\
\text { factor }\end{array}$ \\
\hline 360 & 0.01 & 0.0040 & 2.5 \\
\hline 380 & 0.01 & 0.0058 & 1.7 \\
\hline 400 & 0.01 & 0.0034 & 2.9 \\
\hline 420 & 0.01 & 0.0058 & 1.7 \\
\hline 440 & 0.01 & 0.0034 & 2.9 \\
\hline
\end{tabular}

The variance reduction is less important and varies more for the inputs along the validation window. We think that it is a consequence of the way input variables are defined in the validation problem.

\section{Conclusions and future work}

The results presented in this article are for an adiabatic reactor with a first order kinetic. Similar results have been obtained for different systems including the example described in [1]. Good reductions for variances of state and algebraic variables are obtained in all cases, but for input variables the reductions of the variances are less significant. In the future, we plan to examine the influence of polynomial order used to model the input variables on the variability of the validated uncertainty.

\section{Acknowledgements}

The authors are grateful to the Walloon Region and the European Social funds who co-financed this research.

\section{References}

[1] Liebman, M. J., Edgar, T. F., Lasdon, L. S., Efficient data reconciliation and estimation for dynamic processes using nonlinear programming techniques, Computers \& Chemical Engineering, Vol. 16, № 10/11, 963-986, (1992)

[2] Heyen, G., Maréchal, E., Kalitventzeff, B. (1996). Sensitivity Calculations and Variance Analysis in Process Plant Measurement Reconciliation. Computers and Chemical Engineering 20S, 539-544

[3] Villadsen, J., Michelsen, M. L., Solution of differential equation models by polynomial approximation, Prentice-Hall, Englewood Cliffs, New Jersey, (1978) 